L. A. Cordero

Nagoya Math. J.

Vol. 56 (1974), 29-44

\title{
THE EXTENSION OF $G$-FOLIATIONS TO TANGENT BUNDLES OF HIGHER ORDER
}

\author{
LUIS A. CORDERO*
}

\section{Introduction}

In this paper we describe a canonical procedure for constructing the extension of a $G$-foliation on a differentiable** manifold $X$ to its tangent bundles of higher order and by applying the Bott-Haefliger's construction of characteristic classes of $G$-foliations ([2], [3]) we obtain an infinite sequence $\{\stackrel{0}{\varphi}, \stackrel{1}{\varphi}, \cdots, \stackrel{r}{\varphi}, \cdots\}$ of characteristic classes for those foliations (Theorem 4.8).

By the way, a new equivalence relation between $G$-foliations weaker than the homotopy is defined (Definition 3.7) which we call $r$-homotopy and show that the set of characteristic classes of a $G$-foliation is an invariant of its $r$-homotopy class; some new results in the theory of tangent bundles of higher order are shown (Theorem 1.1 and Lemma 3.10) and the concept of tangent pseudogroup of higher order of a transitive Lie pseudogroup is introduced (Theorem 2.1 and Definition 2.1).

\section{§ 1. Tangent bundles of higher order ([5])}

Let $r \geqslant 0$ be an integer.

Let $M$ be a differentiable $C^{\infty}$ manifold, $\operatorname{dim} M=n$, and let $C^{\infty}(M)$ be the algebra of all differentiable functions on $M$. We denote by $S(M)$ the set of all differentiable maps $\varphi: R \rightarrow M$; we define an equivalence relation on $S(M)$ in the following way: if $\varphi, \psi \in S(M)$ we say $\varphi \widetilde{r} \psi$ if and only if $\varphi(0)=\psi(0)$ and, for every $f \in C^{\infty}(M), f \circ \varphi$ and $f \circ \psi$ have the same $r$-jet in 0 , the origin of $R$; if $\varphi \in S(M),[\varphi]_{r}$ will denote its class of equivalence and if $\varphi(0)=p \in M,[\varphi]_{r}$ is called the $r$-tangent vector

Received November 26, 1973.

Revised June 12, 1974.

* This research was supported by a fellowship of Ministerio de Educacion y Ciencia (Spain) in 1972-73.

** Always differentiable will mean differentiable of class $C^{\infty}$. 
defined by $\varphi$ at the point $p$ of $M$.

Let $\stackrel{r}{T} M$ be the set of all $r$-tangent vectors at all points of $M$; there is a canonical projection

$$
\stackrel{r}{\Pi}_{M}: \stackrel{r}{T} M \rightarrow M
$$

given by $\stackrel{r}{\Pi}_{M}\left([\varphi]_{r}\right)=\varphi(0)$.

In order to define a structure of differentiable manifold on $\stackrel{r}{T} M$, consider a differentiable atlas $\left\{U_{\alpha}, \phi_{\alpha}\right\}_{\alpha \in A}$ of $M$ and let $\left(x_{1}^{\alpha}, \cdots, x_{n}^{\alpha}\right)$ be the coordinate functions on $U_{\alpha}$. On the set $\left(\stackrel{r}{I}_{M}\right)^{-1}\left(U_{\alpha}\right)$ a coordinate system $\left.\stackrel{(\nu) \alpha}{\left(x_{i}\right.}\right), i=1,2, \cdots, n, \nu=0,1, \cdots, r$, is defined by

$$
\stackrel{(\nu) \alpha}{x_{i}}\left([\varphi]_{r}\right)=\frac{1}{\nu !}\left[\frac{d^{\nu}\left(x_{i}^{\alpha}(\varphi(t))\right)}{d t^{\nu}}\right]_{t=0}
$$

for every $[\varphi]_{r} \in\left(\stackrel{r}{\Pi_{M}}\right)^{-1}\left(U_{\alpha}\right)$.

Therefore, $\stackrel{r}{T} M$ is an $n(r+1)$-dimensional differentiable manifold and $\stackrel{r}{\Pi}_{M}$ is a submersion. Besides, for every $p \in M, \stackrel{r}{T}_{p} M=\left(\stackrel{r}{\Pi}_{M}\right)^{-1}(p)$ is canonically diffeomorphic to $\boldsymbol{R}^{r n}$.

$M$ can be canonically imbedded in $\stackrel{r}{T} M$ by taking

$$
i_{M}: M \rightarrow \stackrel{r}{T} M
$$

defined by $i_{M}(x)=\tilde{x}, x \in M$, being $\tilde{x}=\left[\gamma_{x}\right]_{r}$, with $\gamma_{x} \in S(M)$ given by $\gamma_{x}(t)=x$, for every $t \in \boldsymbol{R}$.

Let $N$ be another differentiable manifold and $\phi: M \rightarrow N$ a differentiable map; then, a differentiable map

$$
\stackrel{r}{T} \phi: \stackrel{r}{T} M \rightarrow \stackrel{r}{T} N
$$

is canonically defined by

$$
(\stackrel{r}{T} \phi)\left([\varphi]_{r}\right)=[\phi \circ \varphi]_{r}, \quad \text { for every } \varphi \in S(M) .
$$

Let $M_{0}, M_{1}, M_{2}$ and $M_{3}$ be differentiable manifolds and let

$$
\phi: M_{0} \rightarrow M_{1}, \phi_{1}: \mathrm{M}_{1} \rightarrow M_{2}, \phi^{\prime}: M_{0} \rightarrow M_{2} \text { and } \psi: M_{2} \rightarrow M_{3}
$$

be differentiable maps. Then, it is verified that 


$$
\begin{array}{lc}
\stackrel{r}{T}\left(\phi_{1} \circ \phi\right)=\stackrel{r}{T} \phi_{1} \circ \stackrel{r}{T} \phi, & \stackrel{r}{T}\left(\phi, \phi^{\prime}\right)=\left(\stackrel{r}{T} \phi, \stackrel{r}{T} \phi^{\prime}\right) \\
\stackrel{r}{T}(\phi \times \psi)=\stackrel{r}{T} \phi \times \stackrel{r}{T} \psi, & \stackrel{r}{T} 1_{M}=1_{T M}^{r}
\end{array}
$$

where $1_{M}$ is the identity diffeomorphism and $\stackrel{r}{T}\left(M \times M_{2}\right)$ is canonically identified to $\stackrel{r}{T} M \times \stackrel{r}{T} M_{2}$.

Likewise, if $\phi: M \rightarrow N$ is a submersion (respect. an immersion) $\stackrel{r}{T} \phi$ is also a submersion (respect. an immersion); if $\phi$ is a diffeomorphism, $\stackrel{r}{T} \phi$ is also a diffeomorphism.

If $\phi: M \rightarrow N$ is a differentiable map and $\psi: \stackrel{r}{T} M \rightarrow \stackrel{r}{T} N$ is a differentiable map in such a form that

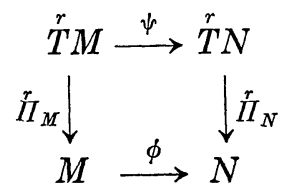

is commutative we shall say " $\psi$ is over $\phi$ "; note that, for each $\phi$, the set of differentiable maps which are over $\phi$ is not empty and let us denote this set $S_{\phi}$.

The following theorem will be important for our purposes and gives a topological relation between a differentiable manifold and its tangent bundles of higher order.

THEOREM 1.1. For every integer $r \geqslant 0, M$ and $\stackrel{r}{T} M$ have the same homotopy type.

Proof. Let $i_{M}$ and $\stackrel{r}{\Pi}_{M}$ as above; it is clear that $\stackrel{r}{\Pi}_{M} \circ i_{M}=1_{M}$.

Now, define a continuous map

$$
\boldsymbol{F}: \stackrel{r}{T} M \times \boldsymbol{R} \rightarrow \stackrel{r}{T} M
$$

by $F\left([\varphi]_{r}, t\right)=\left[\varphi_{t}\right]_{r}$, for $[\varphi]_{r} \in \stackrel{r}{T} M$ and $t \in R$, where $\left[\varphi_{t}\right]_{r} \in \stackrel{r}{T} M$ is defined in the following way: if $\varphi: R \rightarrow M$ defines $[\varphi]_{r}$, we take, for each $t \in \boldsymbol{R}, \varphi_{t}: \boldsymbol{R} \rightarrow M$ given by $\varphi_{t}(s)=\varphi(s(1-t)), \forall s \in \boldsymbol{R}$; it is clear that $\left[\varphi_{t}\right]_{r}$ is well-defined and

$$
\begin{aligned}
& \left.F\right|_{\left.\left.\right|_{T M \times} ^{r} \times\right\}}=1_{T M}^{r} \\
& \left.F\right|_{T_{M} \times\{1\}} ^{r}=i_{M} \circ \stackrel{r}{\Pi}_{M}
\end{aligned}
$$


COROLlaRY 1.1. For every integer $r \geqslant 0$, the de Rham complex $H^{*}(M)$ and $H^{*}(\stackrel{r}{T} M)$ are canonically isomorphic.

\section{§ 2. Tangent pseudogroups of higher order.}

Let $M$ an $n$-dimensional differentiable manifold and let $\stackrel{r}{T} M$ be its tangent bundle of order $r, r \geqslant 0$. Let $G$ be a pseudogroup of local diffeomorphisms of $M$ and consider, for every $g \in G$, the set $S_{g}$ of all local diffeomorphisms of $\stackrel{r}{T} M$ which are over $g$. Then, ${ }^{r} G=\cup_{g \in G} S_{g}$ is a pseudogroup of local diffeomorphisms of $\stackrel{r}{T} M$.

DEFINITION 2.1. We shall call ${ }^{r} G$ the tangent pseudogroup of $G$ of order $r$.

Now, consider the euclidean space $\boldsymbol{R}^{n}$ and its tangent bundle of order $r, \stackrel{r}{T} \boldsymbol{R}^{n}$; for each coordinate open neighborhood $U$ in $\boldsymbol{R}^{n}$ with coordinate functions $\left(x_{1}, \cdots, x_{n}\right)$, consider the coordinate open neighborhood $\stackrel{r}{T} U$ in $\stackrel{r}{T} R^{n}$ and its coordinate functions $\stackrel{(\nu)}{\left(x_{i}\right)}, i=1,2, \cdots, n, \nu=0,1, \cdots, r$, and denote $\varphi^{r}: \stackrel{r}{T} U \rightarrow$ open set $\subset \boldsymbol{R}^{n(r+1)}$ the diffeomorphism defined by the coordinate functions $\stackrel{(\nu)}{x}_{i}$. Let

$$
p_{1}: \boldsymbol{R}^{n} \times \stackrel{r+1}{\cdots} \times \boldsymbol{R}^{n} \rightarrow \boldsymbol{R}^{n}
$$

be the canonical projection onto the first factor; then, every diffeomorphism

$$
\left.\left.\lambda: \varphi^{r} \stackrel{r}{T} U\right) \rightarrow \varphi^{r} \stackrel{r}{T} M\right)
$$

such that $p_{1} \circ \lambda=p_{1}$ defines canonically a differentiable transformation of $\stackrel{r}{T} U$ which is over $1_{U}$.

Now, take $G=\Gamma_{n}$, the Lie pseudogroup of diffeomorphisms on $\boldsymbol{R}^{n}$ (for definition of Lie pseudogroup see [4], p. 36).

THEOREM 2.1. ${ }^{r} \Gamma_{n}$ is a transitive Lie pseudogroup.

Proof. Let $A, B \in \stackrel{r}{T} \boldsymbol{R}^{n}, A \neq B$. We have to show there is ${ }^{r} f \in{ }^{r} \Gamma_{n}$ in such a form that $r f(A)=B$. It may be $\stackrel{r}{\Pi}_{\boldsymbol{R}^{n}}(A)=\stackrel{r}{\Pi}_{\boldsymbol{R}^{n}}(B)$ or $\stackrel{r}{\Pi}_{\boldsymbol{R}^{n}}(A)$ $\neq \stackrel{r}{\Pi}_{\boldsymbol{R}^{n}}(B)$; suppose we are in the second case and put $a=\stackrel{r}{\Pi}_{\boldsymbol{R}^{n}}(A), b$ 
$=\stackrel{r}{\Pi}_{\boldsymbol{R}^{n}}(B)$; then, there exists $f \in \Gamma_{n}$ such that $f(a)=b$ and by using $\stackrel{r}{T} f \in \epsilon^{r} \Gamma_{n}$ we obtain $\stackrel{r}{I}_{R^{n}}((\stackrel{r}{T} f)(A))=\stackrel{r}{I}_{\boldsymbol{R}^{n}}(B)$. Therefore we can restrain us to consider $a=b$.

Thus, let $U \subset \boldsymbol{R}^{n}$ be an open set and $a \in U$; then, $A, B \in \stackrel{r}{T} U$ and put $a^{\prime}=\varphi^{r}(A), b^{\prime}=\varphi^{r}(B), \varphi^{r}$ being the diffeomorphism of $\stackrel{r}{T} U$ on an open set in $\boldsymbol{R}^{n(r+1)}$; clearly, there is a diffeomorphism

$$
\left.\lambda: \varphi^{r}(\stackrel{r}{T} U) \rightarrow \varphi^{r} \stackrel{r}{T} U\right)
$$

in such a form that $\lambda\left(a^{\prime}\right)=b^{\prime}$ and satisfying $p_{1} \circ \lambda=p_{1}$. The differentiable transformation $\eta$ of $\stackrel{r}{T} U$ on itself defined through $\lambda$ is over $1_{U}$ and, therefore, $\eta \in{ }^{r} \Gamma_{n}$; besides, $\eta(A)=B$ and this shows ${ }^{r} \Gamma_{n}$ is transitive.

Now, let $J_{0}^{k}\left({ }^{r} \Gamma_{n}\right)$ be the space of $k$-jets at $\widetilde{0}$ of elements of $r_{n}$, with $\tilde{0}=i_{R^{n}}(0)$ and 0 being the origin of $\boldsymbol{R}^{n}$. Our purpose is to show that $J_{0}^{k}\left(r \Gamma_{n}\right)$ is canonically a differentiable principal bundle over ${ }_{T}^{r} \boldsymbol{R}^{n}$ with group $\left({ }^{r} \Gamma_{n}\right)_{0}^{k}$, the Lie group of $k$-jets of elements of ${ }^{r} \Gamma_{n}$ which keep $\tilde{0}$ fixed.

$\left({ }^{r} \Gamma_{n}\right)_{0}^{k}$ acts freely on $J_{0}^{k}\left(r \Gamma_{n}\right)$ on the right in the natural way: if $j_{0}^{k}(r f) \in\left({ }^{r} \Gamma_{n}\right)_{0}^{k}$ and $j_{0}^{k}(r g) \in J_{0}^{k}\left(r \Gamma_{n}\right)$, then

$$
j_{0}^{k}(r g) \circ j_{0}^{k}(r f)=j_{0}^{k}(r g \circ r f)
$$

is well-defined and if ${ }^{r} g \in S_{g},{ }^{r} f \in S_{f}$, then $\left({ }^{r} g \circ r f f\right) \in S_{\left\langle g^{\circ}, f\right.}$ and, therefore $j_{0}^{k}\left(r g \circ{ }^{r} f\right) \in J_{0}^{k}\left(r_{n}\right)$. In order to obtain the local trivialization of $J_{0}^{k}\left(r \Gamma_{n}\right)$, consider the open covering of $\stackrel{r}{T} \boldsymbol{R}^{n}$ given by $\{\stackrel{r}{T} U\},\{U\}$ being the open sets of $\boldsymbol{R}^{n}$; then, if $p: J_{0}^{k}\left(r_{n} \Gamma_{n}\right) \rightarrow \stackrel{r}{T} \boldsymbol{R}^{n}$ is the canonical projection, for every $U \subset \boldsymbol{R}^{n}$ we define

$$
\left.\phi_{T}^{r} U: p^{-1} \stackrel{r}{T} U\right) \rightarrow \stackrel{r}{T} U \times\left({ }^{r} \Gamma_{n}\right)_{0}^{\frac{c}{d}}
$$

as follows: for every $j_{0}^{k}(r f) \in p^{-1}(\stackrel{r}{T} U)$ with $p\left(j_{0}^{k}(r f)\right)=\tilde{x}$, let ${ }^{r} g_{U} \in{ }^{r} \Gamma_{n}$ such that ${ }^{r} g_{U}(\tilde{0})=\tilde{x}$; then

$$
\phi_{T U}^{r}\left(j_{0}^{k}(r f)\right)=\left(\tilde{x}, j_{\tilde{0}}^{k}\left(\left({ }^{r} g\right)^{-1} \circ^{r} f\right)\right)
$$

Q.E.D.

\section{§3. $r$-extension and $r$-homotopy of foliations.}

Let $M$ be a differentiable manifold and $G$ a pseudogroup of local 
diffeomorphisms acting transitively on $M$; consider the manifold $\stackrel{r}{T} M$ and the tangent pseudogroup ${ }^{r} G$ of order $r$, for every $r \in\{0,1,2, \cdots\}$. We shall suppose from now on that ${ }^{r} G$ is a transitive Lie pseudogroup (that is the case when $M=\boldsymbol{R}^{n}$ and $G=\Gamma_{n}$ as we have shown in theorem 2.1).

Let $X$ be a differentiable manifold, $\operatorname{dim} X \geqslant \operatorname{dim} M$.

Definition 3.1. A G-foliation on $X$ is a maximal family $F$ of submersions

$$
f_{U}: U \rightarrow M
$$

of open sets $U$ in $X,\{U\}$ being an open covering of $X$ and the family $\left\{f_{U}\right\}$ satisfying the following condition: for every $x \in U \cap V$ there exists an element $g_{U V} \in G$ with $f_{U}=g_{U V} \circ f_{V}$ in some vicinity of $x$.

Given a smooth map $f: X^{\prime} \rightarrow X, f$ is transverse to $F$ if the composed maps $f_{U} \circ f$ are submersions; in this case, the maps $f_{U} \circ f$ are the local projections of a $G$-foliation on $X^{\prime}$ called the inverse image $f^{-1} F$ of $F$ via $f$. With this concept, $f$ is called a morphism from $f^{-1} F$ to $F$ and, thus, the $G$-foliations form a category denoted $\mathscr{F}(G)$.

Let $\mathscr{F}\left({ }^{r} G\right)$ be the category of ${ }^{r} G$-foliations.

THEOREM 3.2. Let $F$ be a G-foliation on $X$. There exists, canonically defined, $a^{r} G$-foliation $\stackrel{r}{F}$ on $\stackrel{r}{T} X$ in such a form that the correspondence $F \rightarrow \stackrel{r}{F}$ defines a contravariant functor $\mathscr{R}$ from $\mathscr{F}(G)$ to $\mathscr{F}\left({ }^{r} G\right)$.

Proof. Let $\{U\}$ be the open covering of $X$ and let $\left\{f_{U}\right\}$ be the family of submersions which define the foliation $F$. The ${ }^{r} G$-foliation $\stackrel{r}{F}$ on $\stackrel{r}{T} X$ is defined taking the open covering $\left\{\stackrel{r}{T} U=\left(\stackrel{r}{\Pi}_{X}\right)^{-1}(U)\right\}$ and the family of submersions $\left\{\stackrel{r}{T} f_{U}\right\}$; since this family satisfies the compatibility condition, there exists a maximal family containing it and defining $\stackrel{r}{F}$. Now, let $f: X^{\prime} \rightarrow X$ be a differentiable map which is transverse to $F$. Then, it is clear that $\stackrel{r}{T} f: \stackrel{r}{T} X^{\prime} \rightarrow \stackrel{r}{T} X$ is transverse to $\stackrel{r}{F}$ and it follows $\left(f^{-1} \stackrel{r}{F}\right)$ $=(\stackrel{r}{T} f)^{-1} \stackrel{r}{F}$. The functoriality of the correspondence $F \rightarrow \stackrel{r}{F}$ is shown by a direct computation. 
Definition 3.3. Let $F$ be a $G$-foliation on $X$. The ${ }^{r} G$-foliation $\stackrel{r}{F}$ on $\stackrel{r}{T} X$ defined in theorem 3.2 will be called the r-extension of $F$.

Remark. The construction of Theorem 3.2 is true for every finite positive integer $r$, and, therefore, to each $G$-foliation $F$ on $X$, a sequence $\{\stackrel{0}{F}, \stackrel{1}{F}, \stackrel{2}{F}, \cdots\}$, with $\stackrel{0}{F}=F$, is associated. If $\operatorname{dim} M=m$, that is $\operatorname{codim} F$ $=m$, then $\operatorname{codim} \stackrel{r}{F}=m(r+1)$, for each $r \geqslant 0$.

Let $F_{0}$ and $F_{1}$ be two $G$-foliations on $X$. For each $t \in \boldsymbol{R}$

$$
i_{t}: X \rightarrow X \times R
$$

denotes the canonical injection $x \rightarrow(x, t)$.

Definition 3.4. The $G$-foliations $F_{0}$ and $F_{1}$ are said homotopic, $F_{0} \sim F_{1}$, if there exists a $G$-foliation $F$ on $X \times \boldsymbol{R}$ in such a way that $i_{0}$ and $i_{1}$ are transverse to $F$ and $i_{0}^{-1} F=F_{0}, i_{1}^{-1} F=F_{1}$.

As it is well known, the homotopy of $G$-foliations is an equivalence relation. Denote $\mathscr{H}_{G}(X)$ the set of homotopy class of $G$-foliations on $X$; if $f: X^{\prime} \rightarrow X$ is a morphism of $F, G$-foliation on $X$, to $f^{-1} F, G$-foliation on $X^{\prime}$, it is clear that $f$ defines a map

$$
\mathscr{H}(f): \mathscr{H}_{G}(X) \rightarrow \mathscr{H}_{G}\left(X^{\prime}\right)
$$

and the following theorem is easily proved:

THEOREM 3.5. $\mathscr{H}_{G}(\cdot)$ is a homotopy invariant contravariant functor.

Now, we return to our $r$-extensions.

THEOREM 3.6. Let $F_{0}$ and $F_{1}$ be two homotopic G-foliations on $X$. Then, for every $r \geqslant 0$, their r-extensions $\stackrel{r}{F_{0}}$ and $\stackrel{r}{F_{1}}$ are homotopic ${ }^{r} G$ foliations on $\stackrel{r}{T} X$.

Proof. Let $F$ be the $G$-foliation on $X \times \boldsymbol{R}$ defining the homotopy between $F_{0}$ and $F_{1}$. Consider

$$
\stackrel{r}{T} X \times \boldsymbol{R} \stackrel{1_{T X}^{r} \times i_{R}}{\longrightarrow} \stackrel{r}{T} X \times \stackrel{r}{T} \boldsymbol{R} \stackrel{\sim}{\longrightarrow} T(X \times R) \stackrel{\stackrel{r}{I}_{X \times R}}{\longrightarrow} X \times \boldsymbol{R}
$$

and denote $\lambda=\simeq \circ\left(1_{T X}^{r} \times i_{R}\right)$; then, $\lambda^{-1} \stackrel{r}{F}$ is a ${ }^{r} G$-foliation on $\stackrel{r}{T} X \times \boldsymbol{R}$ which defines a homotopy between $\stackrel{r}{F_{0}}$ and $\stackrel{r}{F_{1}}$; this fact follows from the commutativity of the following diagram, for every $t \in \boldsymbol{R}$, 


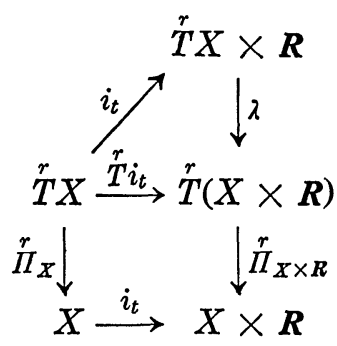

Q.E.D.

Observe that if $F_{0}$ and $F_{1}$ are not homotopic $G$-foliations on $X$, their $r$-extensions could be homotopic, but the converse is an open problem, the answer of which we think to be negative. That leads us to the following definition.

Definition 3.7. Let $r \geqslant 0$ be an integer. Two $G$-foliations $F_{0}$ and $F_{1}$ on $X$ will be said $r$-homotopic, $F_{0} \underset{r}{\sim} F_{1}$, if their $r$-extensions $\stackrel{r}{F}_{0}$ and $\stackrel{r}{F}_{1}$ are homotopic, $\stackrel{r}{F_{0}} \sim \stackrel{r}{F}_{1}$.

PROPOSITION 3.8. $\quad \tilde{r}$ is an equivalence relation.

Remark. The 0-homotopy is the usual homotopy of $G$-foliations and if $F_{0}$ and $F_{1}$ are 0 -homotopic then they are $r$-homotopic for every $r>0$.

Denote, for each $r \geqslant 0, \mathscr{H}_{G}^{r}(X)$ the set of $r$-homotopy classes of $G$ foliations on $X$. Then, we have

THEOREM 3.9. $\quad \mathscr{H}_{G}^{r}(\cdot)$ is a homotopy invariant contravariant functor.

This theorem is a direct consequence of Theorems 3.5 and 3.6 and of the following Lemma.

Lemma 3.10. Let $f_{0}, f_{1}: X^{\prime} \rightarrow X$ two differentiable (differentiably) homotopic maps. Then, for each $r \geqslant 0, \stackrel{r}{T} f_{0}, \stackrel{r}{T} f_{1}: \stackrel{r}{T} X^{\prime} \rightarrow \stackrel{r}{T} X$ are (differentiably) homotopic.

Proof. Let $g: X^{\prime} \times \boldsymbol{R} \rightarrow X$ be the differentiable map defining the homotopy between $f_{0}$ and $f_{1}$. We define a differentiable map

$$
{ }^{r} g: \stackrel{r}{T} X^{\prime} \times R \rightarrow \stackrel{r}{T} X
$$

by ${ }^{r} g=\stackrel{r}{T} g \circ \simeq \circ\left(1_{T^{\prime}}^{r} \times i_{R}\right)$, where $\simeq: \stackrel{r}{T} X^{\prime} \times \stackrel{r}{T} R \rightarrow \stackrel{r}{T}\left(X^{\prime} \times R\right)$ is the can- 
onical diffeomorphism; ${ }^{r} g$ defines actually a homotopy between $\stackrel{r}{T} f_{0}$ and $\stackrel{r}{T} f_{1}$, because for each $t \in \boldsymbol{R}$ the following diagram is commutative:

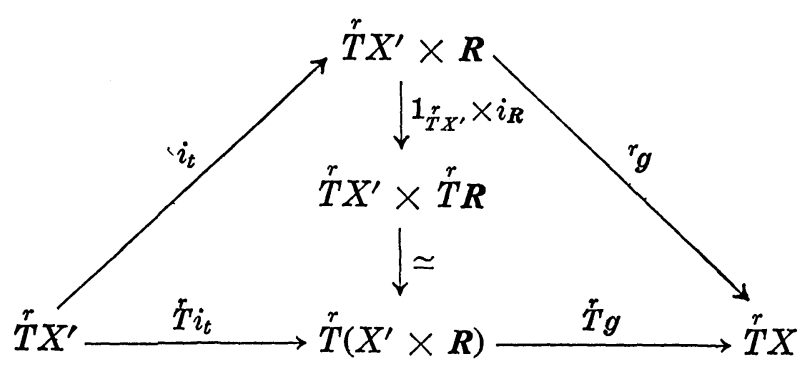

Q.E.D.

\section{§4. Characteristic classes of $\boldsymbol{G}$-foliations.}

Recall briefly the construction of the Bott-Haefliger's characteristic homomorphism for $G$-foliations, following Haefliger ( $\{3\})$.

Let $G$ be a Lie pseudogroup acting transitively on a differentiable manifold $M$; a vector field defined on an open set of $M$ is called a $G$ vector field if the local one parameter group which it generates is in $G$.

Fix a point $0 \in M$; the set of $k$-jets at 0 of $G$-vector fields is a vector space $G^{k}$ which is not a Lie algebra. Then, consider the inverse limit

$$
\underline{G}=\stackrel{\operatorname{Lim}}{\longleftarrow} \underline{G}^{k}
$$

which is a Lie algebra called "the Lie algebra of formal $G$-vector fields". Denote by $A(\underline{G})$ the direct limit of the algebras $A\left(\underline{G}^{k}\right)$ of multilinear alternate forms on $\underline{G}^{k}$; the bracket on $\underline{G}$ induces a differential on $A(\underline{G})$, and we write $H^{*}(\underline{G})$ for the resulting cohomology group.

Denote $J_{0}^{k}(G)$ the space of $k$-jets at 0 of the elements of $G$; this is the total space of a fiber space on $M$; besides, if $G_{0}^{k}$ denotes the Lie group of elements of $J_{0}^{k}(G)$ keeping 0 fixed, $G_{0}^{k}$ acts on $J_{0}^{k}(G)$ on the right and makes it a differentiable principal bundle. Besides, $G$ acts on $J_{0}^{k}(G)$ on the left as a pseudogroup of transformations. Denote $J_{0}^{\infty}(G)$ the inverse limit of the $J_{0}^{k}(G) ; J_{0}^{\infty}(G)$ is endowed with a differentiable structure as follows: a map of a differentiable manifold $X$ on $J_{0}^{\infty}(G)$ is differentiable if its projection on each $J_{0}^{k}(G)$ is differentiable; in this way $J_{0}^{\infty}(G)$ can be looked as a differentiable principal bundle over $M$ 
with group $G_{0}^{\infty}$, the inverse limit of the $G_{0}^{k}$; besides, $G$ acts on $J_{0}^{\infty}(G)$ on the left. We define the algebra $A\left(J_{0}^{\infty}(G)\right)$ of differential forms on $J_{0}^{\infty}(G)$ as the direct limit of the algebras $A\left(J_{0}^{k}(G)\right)$ of differential forms on $J_{0}^{k}(G)$.

THEOREM 4.1 ([3]). $A(\underline{G})$ is canonically isomorphic to the algebra of differential forms on $J_{0}^{\infty}(G)$ which are invariant under the action of $G$ and this isomorphism commutes with the differential operators.

A compact subgroup $K$ of $G_{0}^{\infty}$, playing the role of maximal compact subgroup, is defined being isomorphic to (up to conjugation) the inverse limit of the maximal compact subgroups $K^{s}$ of $G_{0}^{s}$, for each positive integer $s$; the complex $A(\underline{G}, K)$ is the subcomplex of $K$-basic elements of $A(\underline{G})$ and its cohomology algebra will be denoted $H^{*}(\underline{G}, K)$.

THEOREM 4.2 ([3]). Let $F$ be a G-foliation on $X$. There is an algebra homomorphism

$$
\varphi(F): H^{*}(\underline{G}, K) \rightarrow H^{*}(X)
$$

in such a form that if $f: X^{\prime} \rightarrow X$ is transverse to $F$, then

$$
f^{*} \circ \varphi(F)=\varphi\left(f^{-1} F\right)
$$

DEFINITION 4.3. $\operatorname{Im} \varphi(F)$ is called the set of characteristic classes of $F$.

Proposition 4.4. If $F_{0}$ and $F_{1}$ are homotopic G-foliations on $X$, then

$$
\operatorname{Im} \varphi\left(F_{0}\right)=\operatorname{Im} \varphi\left(F_{1}\right) \text {. }
$$

This means that the characteristic classes of a $G$-foliation are invariants of its homotopy class; the following theorem gives a finer characterization.

THEOREM 4.5. Let $F_{0}$ and $F_{1} G$-foliations on $X$. If there is some integer $r \geqslant 0$ such that $F_{0}$ and $F_{1}$ are r-homotopic, then

$$
\operatorname{Im} \varphi\left(F_{0}\right)=\operatorname{Im} \varphi\left(F_{1}\right) \text {. }
$$

This theorem follows from Proposition 4.4 and the following theorem.

THEOREM 4.6. Let $F$ be a G-foliation on $X$ and let $r \geqslant 0$ an integer; if $\varphi(\stackrel{r}{F})$ denotes the Bott-Haefliger's characteristic homomorphism, we have 


$$
\operatorname{Im} \varphi(F)=i_{X}^{*}(\operatorname{Im} \varphi(\stackrel{r}{F}))
$$

where $i_{X}^{*}$ is the isomorphism induced in cohomology by $i_{X}: X \rightarrow \stackrel{r}{T} X$.

To show this theorem, we need a preparatory Lemma. For that, denote $H^{*}\left({ }^{r} \underline{G},{ }^{r} K\right)$ the cohomology of ${ }^{r} K$-basic differential forms on ${ }^{r} \underline{G}$, the Lie algebra of formal ${ }^{r} G$-vector fields; we keep the notations above, only adding the index $r$ in each case.

LEMMA 4.7. Let $r \geqslant 0$ be an arbitrary fixed integer. Let $F$ be $a$ G-foliation on $X$ and let $\stackrel{r}{F}$ be its r-extension. Then:

a) There exists a canonical homomorphism

$$
\sigma: H^{*}(\underline{G}, K) \rightarrow H^{*}\left({ }^{r} \underline{G},{ }^{r} K\right)
$$

such that

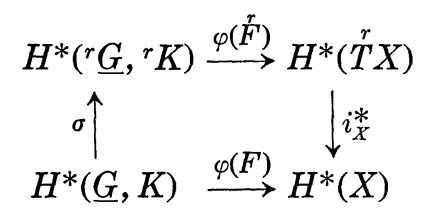

commutes.

b) There exists a canonical homomorphism

$$
\tau: H^{*}\left(\underline{\underline{G}},{ }^{r} K\right) \rightarrow H^{*}(\underline{G}, K)
$$

such that

$$
\begin{gathered}
H^{*}(\underline{r} \underline{G}, r K) \stackrel{\varphi\left(F^{r}\right)}{\longrightarrow} H^{*}(\stackrel{r}{T} X) \\
\tau \downarrow \\
H^{*}(\underline{G}, K) \stackrel{\varphi\left(F^{\prime}\right)}{\longrightarrow} H^{*}(X)
\end{gathered}
$$

commutes.

c) $\tau \circ \sigma=1_{H^{*}(\underline{G}, K)}$ and, hence, $\tau$ is onto.

Proof. 1. Construction of $\sigma$.

Fix the point $\widetilde{0} \in \stackrel{r}{T} M, \widetilde{0}=i_{M}(0)$. Now, consider the map, for each $k \geqslant 0$,

$$
\sigma_{k}: J_{\tilde{0}}^{k}(r G) \rightarrow J_{0}^{k}(G)
$$


defined as follows: let $j_{0}^{k}(r f) \in J_{\tilde{0}}^{k}\left({ }^{r} G\right)$ and let ${ }^{r} f \in{ }^{r} G$ a representative of this jet; then, there is a unique $f \in G$ such that ${ }^{r} f$ is over $f$; we define

$$
\sigma_{k}\left(j_{0}^{k}(r f)\right)=j_{0}^{k}(f)
$$

and $\sigma_{k}$ is, clearly, a well-defined map. Actually, $\sigma_{k}$ induces a homomorphism of Lie groups

$$
\sigma_{k}:{ }^{r} G_{0}^{k} \rightarrow G_{0}^{k}
$$

and, in fact, we get a homomorphism of differentiable principal bundles making commutative the following diagram

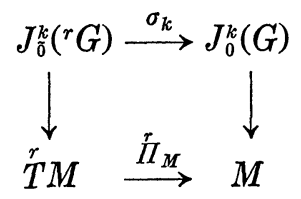

Moreover, if for every ${ }^{r} f \in{ }^{r} G$ with ${ }^{r} f \in S_{f}, f \in G$, we denote $\lambda_{r_{f}}$ (respect. $\lambda_{f}$ ) the differentiable transformation of $J_{0}^{k}(r G)$ (respect. $J_{0}^{k}(G)$ ) defined by the action on the left of ${ }^{r} f$ (respect. $f$ ), a direct computation shows

$$
\lambda_{f} \circ \sigma_{k}=\sigma_{k} \circ \lambda_{r_{f}}
$$

If $\sigma_{k}$ denotes, still, the induced homomorphism between the algebras of differential forms

$$
\sigma_{k}: A\left(J_{0}^{k}(G)\right) \rightarrow A\left(J_{0}^{k}(r G)\right)
$$

the differential forms invariant under the action of $G$ are sent on the differential forms invariant under the action of ${ }^{r} G$. As a consequence, we have canonically a homomorphism

$$
\sigma: A\left(J_{0}^{\infty}(G)\right) \rightarrow A\left(J_{0}^{\infty}\left({ }^{r} G\right)\right)
$$

which induces a new one

$$
\sigma: A(\underline{G}) \rightarrow A\left({ }^{r} \underline{G}\right)
$$

Actually, $\sigma$ induces a homomorphism

$$
\sigma: A(\underline{G}, K) \rightarrow A\left({ }^{r} \underline{G},{ }^{r} K\right)
$$

which induces a homomorphism in cohomology

$$
\sigma: H^{*}(\underline{G}, K) \rightarrow H^{*}\left({ }^{r} \underline{G},{ }^{r} K\right)
$$


In order to prove the commutativity of (4.1) it is sufficient to show the commutativity of

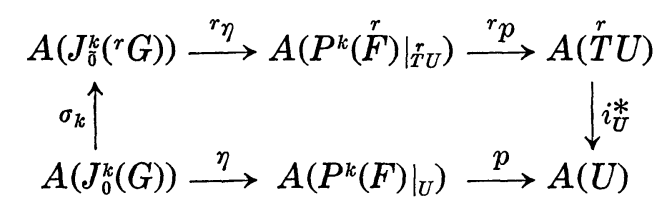

where $U$ is a distinguished open set on $X,\left.P^{k}(F)\right|_{U}$ (respect. $\left.P^{k}(\stackrel{r}{F})\right|_{T U} ^{r}$ ) is the restriction to $U$ (respect. to $\stackrel{r}{T} U$ ) of the principal bundles of $k$-jets of the local projections of $F$ (respect. of $\stackrel{r}{F}$ ); $p$ (respect. ${ }^{r} p$ ) is the homomorphism canonically induced by the local embedding $j_{U}$ (respect. $j_{T U}^{r}$ ) in $\left.P^{k}(F)\right|_{U}$ (respect. $\left.P^{k}\left(\stackrel{r}{F^{\prime}}\right)\right|_{T_{U}} ^{r}$ ) and $\eta$ (respect. ${ }^{r} \eta$ ) is induced by the identification of $J_{0}^{k}(G)$ (respect. $J_{0}^{k}(r G)$ ) to $\left.P^{k}(F)\right|_{U}$ (respect. $\left.P^{k}\left({ }^{r}\right)\right|_{T U} ^{r}$ ) via $f_{U}$ (respect. $\stackrel{r}{T} f_{U}$ ). This diagram, in the limit, and for the $K$-basic $G$-invariant differential forms, induces (4.1).

The embedding $j_{U}:\left.U \rightarrow P^{k}(F)\right|_{U}$ is defined as follows: if $f_{U}: U \rightarrow M$ is the local submersion, for each point $x \in U, j_{U}(x)=j_{0}^{k}\left(g^{-1} f_{U}\right)$, where $g \in G$ verifies $g(0)=f_{U}(x)$, that is, $j_{U}$ is defined through the local trivialization of $P^{k}(F) ; j_{T U}^{r}$ is defined in the same way.

Then, if $\omega \in A\left(J_{0}^{k}(G)\right)$, we have

$$
\left.p(\eta(\omega))\right|_{x}=\left.\eta(\omega)\right|_{j_{0}^{k}\left(g-1 f_{U}\right)}=\left.\omega\right|_{j_{0}^{k}(g)}
$$

and, if $\tilde{x}=i_{U}(x)$

$$
\begin{aligned}
& \left.i_{U}^{*}\left({ }^{r} p\left({ }^{r} \eta\left(\sigma_{k}(\omega)\right)\right)\right)\right|_{\alpha}=\left.{ }^{r} p\left({ }^{r} \eta\left(\sigma_{k}(\omega)\right)\right)\right|_{\tilde{x}} \\
& \quad=\left.{ }^{r} \eta\left(\sigma_{k}(\omega)\right)\right|_{\left.j \tilde{o}\left(r^{r} g\right)-1 T^{r} f_{U}\right)}=\left.\sigma_{k}(\omega)\right|_{j_{\tilde{0}}^{k}(T)}=\left.\omega\right|_{j_{0}^{k}(g)}
\end{aligned}
$$

Hence, (4.3) commutes.

2. Construction of $\tau$.

For each $k \geqslant 0$, we define a differentiable map

$$
\tau_{k}: J_{0}^{k}(G) \rightarrow J_{\tilde{0}}^{k-r}\left({ }^{r} G\right)
$$

by $\tau_{k}\left(j_{0}^{k}(f)\right)=j_{0}^{k-r}(\stackrel{r}{T} f)$ for $f \in G$, if $k>r$, and $\tau_{k}\left(j_{0}^{k}(f)\right)=j_{0}^{0}(\stackrel{r}{T} f)$ if $k \leqslant r$. It is clear that $\tau_{k}$ is a well-defined differentiable map and it induces a homomorphism

$$
\tau_{k}: A\left(J_{\overline{0}}^{k-r}\left({ }^{r} G\right)\right) \rightarrow A\left(J_{0}^{k}(G)\right)
$$


and, in the limit, we have the homomorphism

$$
\tau: A\left(J_{\tilde{0}}^{\infty}\left({ }^{r} G\right)\right) \rightarrow A\left(J_{0}^{\infty}(G)\right) .
$$

As above, $\tau$ sends the differential forms invariant under the action of ${ }^{r} G$ on differential forms invariant under the action of $G$, because

$$
\lambda_{T f}^{r} \circ \tau_{k}=\tau_{k} \circ \lambda_{f}
$$

for every $k \geqslant 0$. Hence, $\tau$ defines a homomorphism

$$
\tau: A\left({ }^{r} \underline{G}\right) \rightarrow A(\underline{G}) \text {. }
$$

Obviously, for each $k \geqslant 0, \tau_{k}$ defines a homomorphism of differentiable principal bundles, making commutative the following diagram

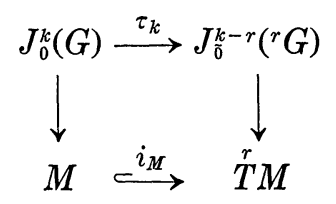

and, in fact, $\tau$ induces a homomorphism in cohomology

$$
\tau: H^{*}\left({ }^{r} \underline{G},{ }^{r} K\right) \rightarrow H^{*}(\underline{G}, K) .
$$

The commutativity of (4.2) follows from the commutativity of

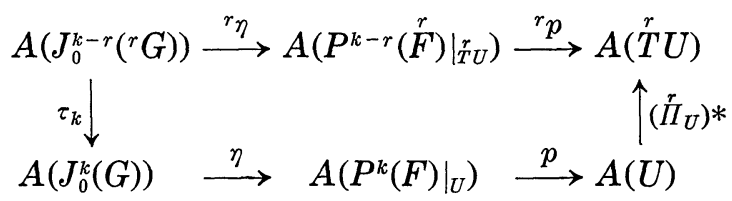

because if $\omega \in A\left(J_{\tilde{0}}^{k-r}\left({ }^{r} G\right)\right)$ and $\tilde{x} \in \stackrel{r}{T} U$ with $\stackrel{r}{I}_{U}(\tilde{x})=x$, we have

$$
\left.{ }^{r} p\left({ }^{r} \eta(\omega)\right)\right|_{\tilde{x}}=\left.{ }^{r} \eta(\omega)\right|_{j_{\tilde{0}}^{k-r}\left((r g)-1 \tilde{T}_{U}\right)}=\left.\omega\right|_{j_{\tilde{0}}^{k-r}\left(r^{r} g\right)}
$$

and

$$
\begin{aligned}
& \left.\left(\stackrel{r}{I}_{U}\right) *\left(p\left(\eta\left(\tau_{k}(\omega)\right)\right)\right)\right|_{\tilde{x}}=\left.p\left(\eta\left(\tau_{k}(\omega)\right)\right)\right|_{x} \\
& \quad=\left.\eta\left(\tau_{k}(\omega)\right)\right|_{j_{0}^{k}\left(g-1 f^{k}\right)}=\left.\tau_{k}(\omega)\right|_{j_{0}^{k}(g)}=\left.\omega\right|_{j_{\tilde{0}}^{k-r}\left(T^{k} g\right)}
\end{aligned}
$$

but ${ }^{r} g \in S_{g}$ and, by definition of $j_{T U}^{r}$ it is ${ }^{r} g=\stackrel{r}{T} g$ and we have the commutativity of (4.4).

3. $\tau \circ \sigma=1_{H^{*}(\underline{G}, K)}$

For that, it is sufficient to show that 


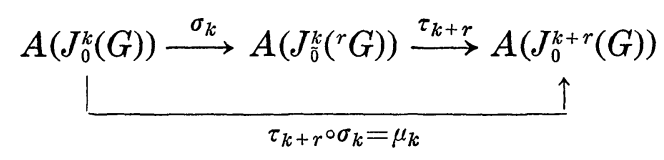

induces the identity in the limit. Then, consider, for each $k>0$,

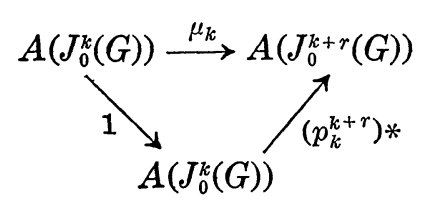

where $1=1_{A\left(J_{0}^{k}(G)\right)}$ and

$$
p_{k}^{k+r}: J_{0}^{k+r}(G) \rightarrow J_{0}^{k}(G)
$$

is the canonical projection. But (4.5) commutes because the following diagram

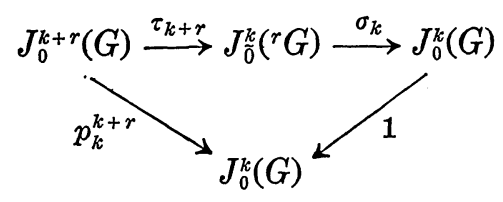

commutes trivially.

The assertion, now, follows from the commutativity of (4.5).

Proof of Theorem 4.6. (4.1) implies

$$
i_{X}^{*}\left(\operatorname{Im} \varphi\left(F^{\prime}\right)\right) \supseteq \operatorname{Im} \varphi(F)
$$

and (4.2) implies

$$
\left(\stackrel{r}{I}_{X}\right)^{*}(\operatorname{Im} \varphi(F)) \supseteq \operatorname{Im} \varphi\left(\stackrel{r}{F}^{\prime}\right)
$$

because $\tau$ is onto. Then, as $i_{X}^{*} \circ\left(\stackrel{r}{I}_{X}\right)^{*}=1_{H^{*}(X)}$, we obtain

$$
\operatorname{Im} \varphi(F)=i_{X}^{*}(\operatorname{Im} \varphi(\stackrel{r}{F}))
$$

Q.E.D.

Finally, combining the Bott-Haefliger's result (theorem 4.2), their definition of characteristic class of a $G$-foliation and our results, we can assert:

THEOREM 4.8. Let $\mathscr{F}(G)$ the category of G-foliations; there exists 
an infinite sequence $\{\stackrel{0}{\varphi}, \stackrel{1}{\varphi}, \cdots, \stackrel{r}{\varphi}, \cdots\}$ of characteristic classes of $G$ foliations, that is, natural transformations

$$
\stackrel{r}{\varphi}: \mathscr{F}(G) \rightarrow H^{*}(; \boldsymbol{R})
$$

satisfying

$$
\stackrel{r}{\varphi}\left(f^{-1} F\right)=f^{*} \stackrel{r}{\varphi}(F)
$$

and $\stackrel{0}{\varphi}$ being the Bott-Haefliger's characteristic class.

Proof. Define, for a $G$-foliation $F, \stackrel{r}{\varphi}(F)=\varphi\left(\stackrel{r}{F^{\prime}}\right)$, and apply the above theorem.

Q.E.D.

\section{REFERENCES}

[1] Bott, R., Lectures on characteristic classes and foliations. Lect. Notes on Math., Springer, $\mathrm{n}^{\circ} 279,1972$.

[2] Bott, R. and Haefliger, A., On characteristic classes of $\Gamma$-foliations. Bull. Am. Math. Soc., 1973.

[ 3 ] Haefliger, A., Sur les classes caractéristiques des feuilletages. Sem. Bourbaki, exposé 412, 1971/72.

[ 4 ] Kobayashi, S., Transformation groups in differential geometry. Springer-Verlag 1972.

[5] Morimoto, A., Prolongation of $G$-structures to tangent bundles of higher order. Nagoya Math. J., 38 (1970), 153-179.

Departamento de Geometria y Topologia

Universidad de Santiago de Compostela 